\title{
Bumping Phenomenon During Continuous Coagulation With Bipolar Forceps
}

\author{
Takeshi MIKAMI, ${ }^{1}$ Masahiko WANIBUCHI, ${ }^{1}$ and Nobuhiro MIKUNI ${ }^{1}$ \\ ${ }^{1}$ Department of Neurosurgery, Sapporo Medical University, Sapporo, Hokkaido
}

\begin{abstract}
During continuous bipolar coagulation, the coagulum sometimes ruptures suddenly and audibly, leading to hemostasis failure and damage to the surrounding tissues. Such coagulum rupture is a phenomenon that is similar to microwave bumping, which occurs because of unequal heat distribution as a result of sudden elevation in temperature. The present study investigated the conditions under which bumping can occur during bipolar coagulation using samples of whole blood. Two types of bipolar forceps were assessed: forceps with gold-plated tips and IsoCool $^{\mathrm{TM}}$ forceps. The time until the bumping phenomenon occurred was measured, and the occurrence rate of bumping was calculated. Thermal characteristics were then assayed using a thermographic camera. As the output of the bipolar coagulator increased, the time until bumping decreased whereas the occurrence rate of bumping during coagulation increased. At lower outputs, the occurrence rate of bumping was significantly lower using IsoCool $^{\mathrm{TM}}$ forceps than that using forceps with gold-plated tips $(p<0.0001)$. Furthermore, bumping during coagulation could be prevented if the tips of the forceps at the coagulation site were repeatedly opened and closed. The bumping phenomenon is influenced by various factors such as heat, electrical power, equipment, and coagulation technique. Neurosurgeons should understand the technical certain aspects of these surgical tools and develop appropriate advanced techniques for temperature control.
\end{abstract}

Key words: bipolar forceps, bumping, coagulum, neurosurgical procedure, thermography

\section{Introduction}

Bipolar coagulation forceps are an invaluable tool for modern neurosurgical operations. ${ }^{7,12,19)}$ Technological advances have led to significant improvements in bipolar forceps, enabling effective bleeding control and fine dissection. ${ }^{1-5,10,11,13-18,20)}$ Nevertheless, the use of this type of forceps remains associated with certain problems such as adherence of charred tissues to the forceps; this problem sometimes leads to hemostasis failure and damage to the surrounding tissues. ${ }^{2,13,14)}$ In addition, continuous coagulation or coagulation under relatively high electrical output sometimes causes the coagulum to explode or rupture audibly. This sudden localized bursting or "bumping" of the coagulum is considered to result from expansion due to unequal heat distribution that occurs because of sudden temperature elevation. This bumping phenomenon is considered highly undesirable because it can complicate or delay the hemostasis of tiny or otherwise fragile

Received October 19, 2011; Accepted December 21, 2011 vessels, such as those that are encountered in the deep white matter around the nidus in patients with arteriovenous malformation or in vascular-rich tumors.

The bumping phenomenon that occurs during coagulation is similar to microwave bumping which occurs during heating in a microwave oven. Microwave bumping is an explosion phenomenon that is characterized by the jostling or shaking of the food container and is usually accompanied by an abrupt and explosive sound. ${ }^{5}$ This phenomenon is also usually accompanied by the splattering of liquid and food particles on the inside surface of the microwave oven. Although coagulum bumping during bipolar coagulation is common in clinical practice, no previously published studies have described its characteristics.

The present study investigated the bumping phenomenon during coagulation using two different types of forceps and coagulators to examine the factors influencing the bumping phenomenon during coagulation in neurosurgical operations. 

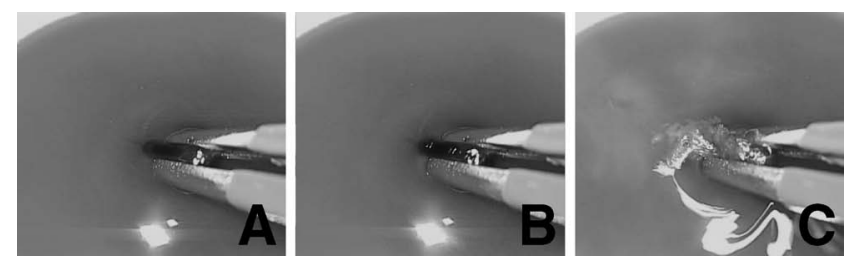

Fig. 1 Photographs of the bumping phenomenon during coagulation. Coagulation was performed using goldplated forceps at an electric power of 20 Malis units (3 W). Coagulum was formed through continuous coagulation (A: at 3.0 seconds, B: at 5.9 seconds), and burst audibly (C: at 6.0 seconds).

\section{Materials and Methods}

Two types of bipolar forceps were evaluated, both of which had 1-mm-wide tips: one type of forceps had tips plated with $50 \mu \mathrm{m}$ gold (KH-2001; Codman/ Johnson \& Johnson K.K., Tokyo); the other was made using heat pipe technology (IsoCool ${ }^{\mathrm{TM}}$; Codman \& Shurtleff, Inc., Raynham, Massachusetts, USA). Before use, the bipolar forceps were washed with deionized water and ethanol, and then dried at $50^{\circ} \mathrm{C}$. Coagulation was performed using a Malis bipolar coagulator (CMC-III; Codman \& Shurtleff, Inc.) and a Vesalius bipolar coagulator (MCN; Telea Electronic Engineering S.r.l., Vicenza, Italy).

In vitro assay of bumping used human whole blood, because in clinical situations most of the coagulum adhering to the tips of bipolar forceps consists of blood. The methods were previously described in detail. ${ }^{13,14)}$ Figure 1 illustrates an example of the bumping phenomenon, which occurred when coagulum formed through continuous coagulation burst audibly. In vitro assay was performed to measure elapsed time prior to coagulum bursts. A sample of $100 \mu \mathrm{l}$ of human whole blood was inoculated on a glass slide and the tips of the bipolar forceps (goldplated or IsoCool ${ }^{\mathrm{TM}}$ ) placed in the blood sample, with a distance of $1 \mathrm{~mm}$ between the tips. Coagulation was performed using a Malis bipolar coagulator at various electric power settings (15-40 Malis units; 1.6-11.0 W). The time until the bumping phenomenon occurred was measured. Coagulation was also performed using a Vesalius bipolar coagulator (5-30 Vesalius units; 0-22.0 W). The time until the bumping phenomenon occurred was measured. Each type of forceps was used for 10 iterations.

Similarly, the occurrence rate of the bumping phenomenon was assessed during coagulation with both types of bipolar forceps. A sample of $100 \mu \mathrm{l}$ of human whole blood was inoculated on a glass slide and the tips of the bipolar forceps (gold-plated or
IsoCool $^{\mathrm{TM}}$ ) placed in the blood sample, with a distance of $1 \mathrm{~mm}$ between the tips. Electrical power was applied at various levels (10-40 Malis units; 1.0-11.0 W) for a constant duration (15 seconds) to induce coagulation. This procedure was repeated 50 times under each condition, and the probabilities that bumping phenomenon would occur were calculated for the two conditions. The probability that bumping would occur was defined as the occurrence rate of bumping.

Thermographic study measured the temperature of the coagulum at the tips of the bipolar forceps at 15-second intervals of coagulation. Coagulation was discontinued if the bumping phenomenon occurred. A sample of $100 \mu \mathrm{l}$ of human whole blood was inoculated on a glass slide and the tips of the bipolar forceps (gold-plated or IsoCool ${ }^{\mathrm{TM}}$ ) placed on the blood sample, with a distance of $1 \mathrm{~mm}$ between the tips. Electrical power was applied at a constant level (20 Malis units; 1.6 W) for 15 seconds. Coagulation was first performed with the tips in a fixed position). Subsequently, coagulation was performed while repeatedly opening and closing the tips of the goldplated forceps. The temperature was measured using an infrared thermography camera (TH9100MR; NEC Avio Infrared Technologies Co., Ltd., Tokyo) and analyzed using the software provided with the camera (Report Generator NS9200; NEC Avio Infrared Technologies Co., Ltd.).

In this experimental study, we applied electrical power at higher levels and for longer durations than would ordinarily be used in a clinical setting. These exaggerated conditions facilitated better understanding of the different rates of coagulation that result when different materials are used.

Data are expressed as means \pm standard deviations. The data on the elapsed time before bumping occurred were analyzed using simple regression analysis. The occurrence rate of bumping was quantified with an estimated 95\% confidence interval and compared between the two types of forceps using the chi-square test for independence. A probability value of less than 0.05 was considered statistically significant.

\section{Results}

In vitro assay of bumping showed that the mean elapsed time prior to bumping decreased as the output increased, as illustrated in Fig. 2. Regression analysis for the data collected using the Malis bipolar coagulator found that the regression line for the gold-plated forceps was $\mathrm{y}=19.216 \mathrm{x}^{-1.2663} ; \mathrm{R}=$ 0.948 ( $p<0.0001$ ). This tendency was confirmed in the data using the Vesalius bipolar coagulator. 


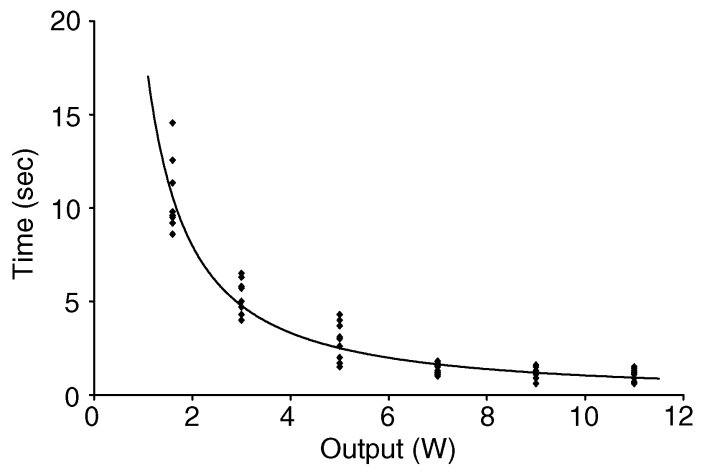

Fig. 2 Graph relating power output to coagulation time achieved prior to the bumping phenomenon. Each value represents the mean of 10 coagulation procedures. The regression line was calculated as $y=19.216 x^{-1.2663} ; R=$ $0.948(p<0.0001)$.

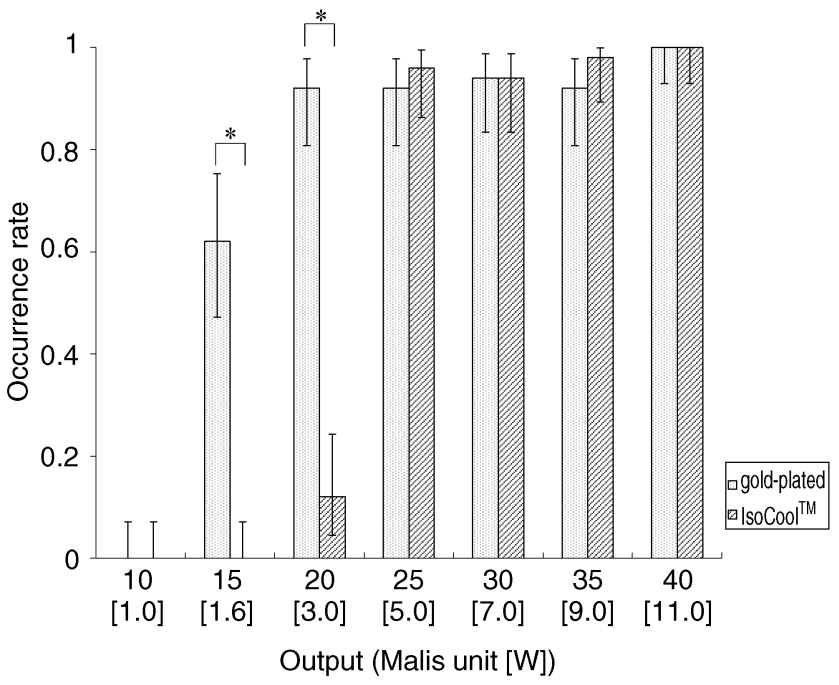

Fig. 3 Graph relating power output to the occurrence rate of bumping during coagulation using the Malis bipolar coagulator according to the type of bipolar forceps used. Values represent the occurrence rate of bumping. There are significant differences between the forceps types at lower output levels (15-20 Malis units; 1.6-3.0 W). ${ }^{*} \mathbf{p}<0.05$.

The occurrence rate of bumping increased as the output increased, as depicted in Fig. 3. At lower output levels using the Malis bipolar coagulator (15-20 Malis units; $1.6-3.0 \mathrm{~W}$ ), the occurrence rate of bumping using the IsoCool ${ }^{\mathrm{TM}}$ forceps was significantly lower than that using the gold-plated forceps ( $p<0.0001$ ) (Fig. 3). This tendency was confirmed using the Vesalius bipolar coagulator, as depicted in Fig. 4.

The results of the thermal analysis of the tips of the bipolar forceps are depicted in Fig. 5. The tem-

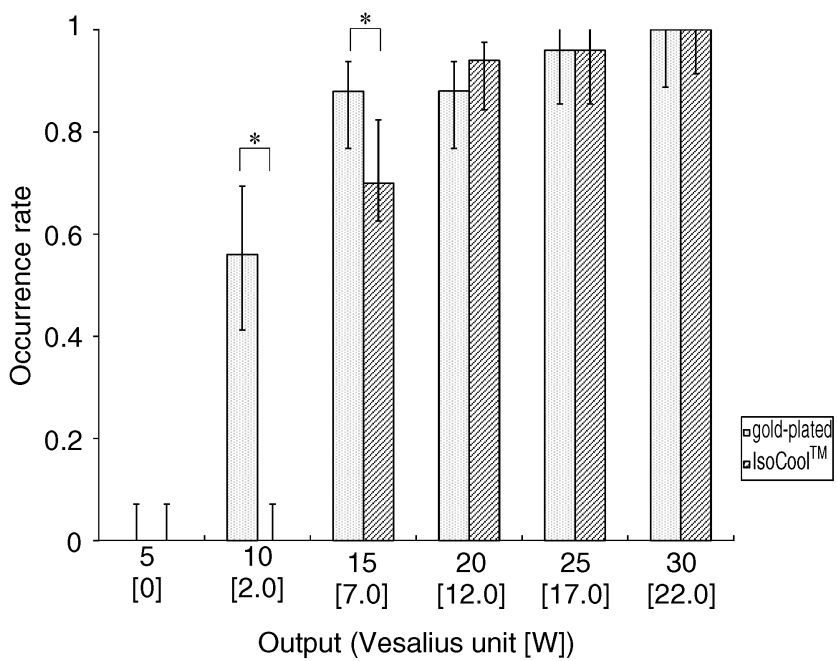

Fig. 4 Graph relating power output to the occurrence rate of bumping during coagulation using the Vesalius bipolar coagulator according to the type of bipolar forceps used. Values represent the occurrence rate of bumping. There are significant differences between the forceps types at lower output levels (10-15 Vesalius units; 2.0-7.0 W). ${ }^{*} \mathbf{p}<0.05$.

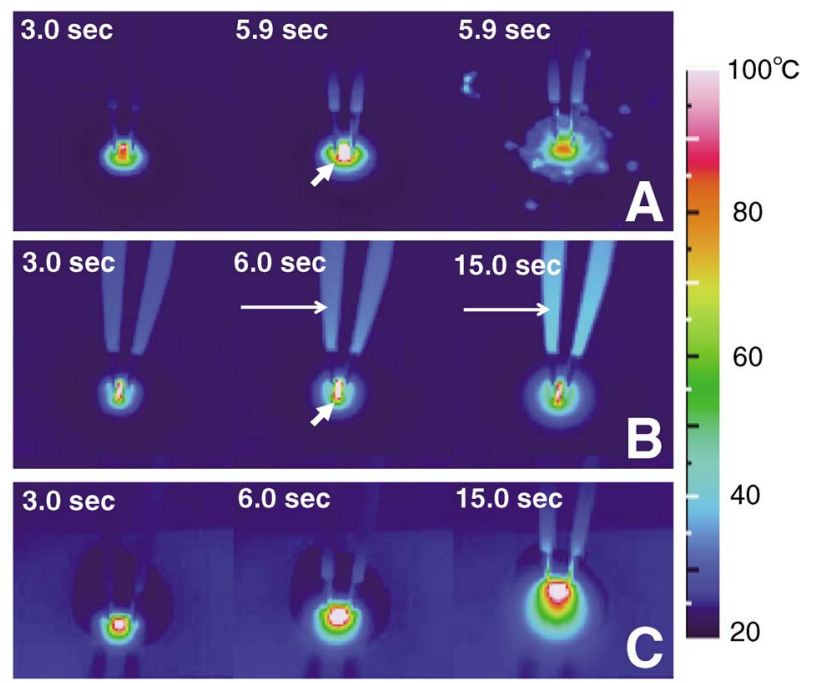

Fig. 5 Photographs of the thermograph during coagulation. The timeline is indicated at the upper left. A: With the gold-plated forceps, the bumping phenomenon had not occurred at 5.9 seconds after the start of coagulation, but the area exceeding $100^{\circ} \mathrm{C}$ at the tips (short arrow) became larger. Bumping occurred at 6 seconds. B: With IsoCool ${ }^{\mathrm{TM}}$ forceps, the area exceeding $100^{\circ} \mathrm{C}$ at the tips (short arrow) was smaller than that of the gold-plated forceps at 6 seconds. The shaft temperatures elevated during coagulation (long arrows), and 15 seconds of coagulation was achieved without bumping. C: When the gold-plated forceps were repeatedly opened and closed during coagulation, the temperature of the tips could be maintained at around $100^{\circ} \mathrm{C}$, and 15 seconds of coagulation was achieved without bumping. 
peratures at the tips of the fixed gold-plated forceps rose to $100^{\circ} \mathrm{C}$ or higher, and the portion exceeding $100^{\circ} \mathrm{C}$ subsequently became larger (Fig. 5A). Bumping had not yet occurred at 5.9 seconds. Bumping occurred after 6 seconds: the coagulum was splashed and the temperatures at the tips fell. As for the IsoCool ${ }^{\mathrm{TM}}$ forceps, the area exceeding $100^{\circ} \mathrm{C}$ at the tips was smaller than that of the gold-plated forceps, allowing 15 seconds of continuous coagulation without bumping (Fig. 5B). The area exceeding $100^{\circ} \mathrm{C}$ at the tips remained small. The shaft temperatures of the IsoCool ${ }^{\mathrm{TM}}$ forceps were observed to elevate during coagulation, implying that heat diffusion was occurring in the direction of the shaft. When the gold-plated forceps were repeatedly opened and closed during coagulation as depicted in Fig. 5C, the temperature of the tips could be maintained at around $100^{\circ} \mathrm{C}$, and continuous coagulation could be achieved for 15 seconds without coagulum bumping occurring.

\section{Discussion}

The bumping phenomenon has previously been thermodynamically investigated. ${ }^{8,9)}$ Bumping frequently occurs when liquids are heated in smooth-walled containers; the absence of irregularities on the internal surfaces of these containers hinders the formation of bubbles, so superheating occurs and the temperature of the liquid rises above its boiling point without formation of vapor. Although the vapor pressure exceeds the atmospheric pressure, the liquid is prevented from boiling because bubble formation is suppressed, so the bubbles which finally form subsequently burst suddenly and violently as the excess vapor pressure built up at that location in the liquid is finally released. In other words, bumping occurs because of irregular distribution of heat. Perhaps the best-known example of bumping is microwave bumping, ${ }^{6)}$ but a similar phenomenon sometimes occurs during the coagulation procedure. This phenomenon is different from electrical sparking. ${ }^{11)}$ Especially in patients with arteriovenous malformation, the small, fragile vessels in the deep white matter around the nidus are frequently subjected to bumping during continuous coagulation. When heat is continuously applied to these small and fragile vessels for too long, they occasionally burst open, thus hindering effective bleeding control. Therefore, avoiding the bumping phenomenon and controlling bleeding in such fragile vessels is very important. To prevent the occurrence of bumping, the effects of various levels of electrical power and the technical aspects of the devices used for coagulation need to be understood clearly.
Rapid heating of liquids in containers made of metals with high heat conductivity (such as stainless steel) leads to an irregular distribution of heat, which results in the bumping phenomenon. Temperature control is the most important factor in ensuring adequate coagulation without bumping. Forceps made using heat pipe technology, such as the IsoCool ${ }^{\mathrm{TM}}$ forceps, feature a built-in cooling system. ${ }^{20)}$ based on heat-conducting pipes made of tiny copper spheres which are compressed into a wicking structure under vacuum. Using this type of forceps as opposed to ordinary gold-plated forceps, continuous coagulation can be applied for longer periods without bumping because the heat-pipe forceps maintain lower temperatures during coagulation. However, if the output is too high, bumping occurs regardless of which bipolar forceps and bipolar coagulator are used. Therefore, we do not think that the IsoCool ${ }^{\mathrm{TM}}$ forceps offer superior performance in all situations. Based on clinical experience and this investigation, IsoCool ${ }^{\mathrm{TM}}$ forceps appear to be effective at allowing continuous coagulation for bleeding such as oozing in which tumors are situated away from important structures.

Especially when coagulation is performed at vital sites, the electrical power and the duration of application should be minimized. Although the temperature should be high enough to ensure that coagulation is successful, ideally, it should also be low enough to prevent tissue adherence to the forceps and bumping. In practice, the optimal temperature may be approximately $80^{\circ} \mathrm{C}$.4) Several methods for controlling the temperature are available, including irrigation systems, ${ }^{20}$ ) but the keys to this procedure are not only the equipment used but also the technique applied, as our results have demonstrated. Specifically, heat distribution in the tissue can be made more even by repeatedly opening and closing the forceps tips during coagulation. Furthermore, it is essential to coagulate in a short time or intermittently for situations that require pinpoint coagulation, taking thermodynamics into consideration. These findings improve our understanding of coagulation and will result in safer and more sophisticated neurosurgical procedures.

\section{Acknowledgments}

We thank Yoshiro Kubo and Osho Kimura, Codman/Johnson \& Johnson K.K., for their technical support in the preparation of the bipolar forceps.

\section{Disclaimer}

None of the authors have a financial interest in the 
bipolar forceps used in this study.

\section{References}

1) Bergdahl B, Vallfors B: Studies on coagulation and the development of an automatic computerized bipolar coagulator. Technical note. J Neurosurg 75: 148151,1991

2) Casotto A, Piattelli M: A new device for electrocoagulation of small vessels. Acta Neurochir (Wien) 71: 151-160, 1984

3) Ceviker N, Keskil S, Baykaner K: A new coated bipolar coagulator: technical note. Acta Neurochir (Wien) 140: 619-620, 1998

4) Dujovny M, Dujovny N, Gundamraj NR, Misra M: Bipolar coagulation in neurosurgery. Surg Neurol 49: 328-332, 1998

5) Dujovny M, Vas R, Osgood CP, Maroon JC, Janetta PJ: Automatically irrigated bipolar forceps. Technical note. J Neurosurg 43: 502-503, 1975

6) $\mathrm{Fu}$ YC, Tong $\mathrm{CH}$, Lund DB: Microwave bumping: quantifying explosions in foods during microwave heating. J Food Sci 59: 899-904, 1994

7) Greenwood J: Two-point coagulation. A new principle and instrument for applying coagulation current in surgery. Am J Surg 50: 267-270, 1940

8) Helrich CS: Modern Thermodynamics with Statistical Mechanics. Berlin, Heidelberg, Springer-Verlag, 2008

9) Huang K: Statistical Mechanics, ed 2. West Sussex, John Wiley \& Sons, 1987

10) Jacques S, Bullara LA, Pudenz RH: Microvascular bipolar coagulator. Technical note. J Neurosurg 44: 523-524, 1976

11) Malis LI: Electrosurgery. Technical note. J Neurosurg 85: 970-975, 1996

12) Malis LI: Electrosurgery and bipolar technology.
Neurosurgery 58: ONS1-12, 2006

13) Mikami T, Minamida Y, Koyanagi I, Houkin K: Novel bipolar forceps with protein repellence using goldpolytetrafluoroethylene composite film. Neurosurgery 60: ONS157-160, 2007

14) Mikami T, Takahashi A, Hashi K, Gasa S, Houkin K: Performance of bipolar forceps during coagulation and its dependence on the tip material: a quantitative experimental assay. Technical note. J Neurosurg 100: 133-138, 2004

15) Ohta T, Funatsu N, Kuroiwa T, Matsui T: Bipolar diathermy forceps with automatic irrigation. Technical note. J Neurosurg 62: 933-934, 1985

16) Sakatani K, Ohtaki M, Morimoto S, Hashi K: Isotonic mannitol and the prevention of local heat generation and tissue adherence to bipolar diathermy forceps tips during electrical coagulation. Technical note. J Neurosurg 82: 669-671, 1995

17) Samii A, Dujovny M: Kirwan “non-stick” bipolar forceps. Surg Neurol 45: 297-298, 1996

18) Sugita K, Tsugane R: Bipolar coagulator with automatic thermocontrol. Technical note. J Neurosurg 41: 777-779, 1974

19) Vallfors B, Erlandson BE, Wieck BO, Hansson HA, Svensson J: Coagulation in neurosurgery. Acta Neurochir (Wien) 55: 29-34, 1980

20) Vellimana AK, Sciubba DM, Noggle JC, Jallo GI: Current technological advances of bipolar coagulation. Neurosurgery 64: 11-19, 2009

Address reprint requests to: Takeshi Mikami, MD, Department of Neurosurgery, Sapporo Medical University, South 1 West 16, Chuo-ku, Sapporo 060-8543, Japan. e-mail: tmikami@sapmed.ac.jp 